\title{
Histologic evaluation of critical size defect healing with natural and synthetic bone grafts in the pigeon (Columba livia) ulna
}

\begin{abstract}
Fracture and bone segment loss are major clinical problems in birds. Achieving bone formation and clinical union in a fracture case is important for the survival of the bird. To evaluate the efficacy of bone grafts for defect healing in birds, 2 different bone grafts were investigated in the healing of a bone defect in 24 healthy pigeons (Columba livia). In each bird, a 1-cm critical size defect (CSD) was created in the left ulna, and the fracture was stabilized with external skeletal fixation (ESF). A graft of hydroxyapatite (HA) alone ( $\mathrm{n}=12$ birds) or demineralized bone matrix (DBM) combined with HA ( $\mathrm{n}=12$ birds) was implanted in the CSD. The CSD healing was evaluated at 3 endpoints: 3, 6, and 12 weeks after surgery. Four birds were euthanatized at each endpoint from each treatment group, and bone graft healing in the ulna CSD was evaluated by histologic examination. The CSD and graft implants were evaluated for quality of union, cortex development, and bone graft incorporation. Results showed no graft rejection in any bird, and all birds had connective tissue formation in the defect because of the bone graft application. These results suggest that bone defect healing can be achieved by a combination of osteoinductive and osteoconductive bone graft materials for clinical union and new bone regeneration in birds. The combination of DBM and HA resulted in a better quality bone graft $(\mathrm{P}<.05)$ than did HA alone, but there was no significant differences in cortex development or bone graft incorporation at 3, 6, or 12 weeks. From the results of this study, we conclude that HA bone grafts, alone or in combination with DBM, with external skeletal fixation is suitable and safe for bone defect and fracture treatment in pigeons.
\end{abstract}

Keyword: Avian; Columba livia; Critical size defect; Demineralized bone matrix; Histology; Hydroxyapatite; New bone formation; Pigeon; Ulna 